Editor's Note: These short reviews of a recent paper in the Journal, written exclusively by graduate students or postdoctoral fellows, are intended to mimic the journal clubs that exist in your own departments or institutions. For more information on the format and purpose of the Journal Club, please see http://www.jneurosci.org/misc/ifa_features.shtml.

\title{
Dorsolateral Prefrontal Cortex, Working Memory, and Prospective Coding for Action
}

\author{
Rogier B. Mars ${ }^{1,2}$ and Meike J. Grol ${ }^{3,4}$ \\ ${ }^{1}$ Sobell Department of Motor Neuroscience and Movement Disorders, Institute of Neurology and ${ }^{2}$ Institute of Cognitive Neuroscience, University College \\ London, London WC1N 3AR, United Kingdom, ${ }^{3}$ Helmholtz Research Institute, Experimental Psychology, Utrecht University, 3584 CS Utrecht, The \\ Netherlands, and ${ }^{4}$ F.C. Donders Centre for Cognitive Neuroimaging, Radboud University Nijmegen, 6500 HB Nijmegen, The Netherlands \\ Review of Procyk and Goldman-Rakic (http://www.jneurosci.org/cgi/content/full/26/44/11313)
}

The role of the dorsolateral prefrontal cortex (DLPFC) in the control of behavior remains a topic of ongoing controversy. The discovery of sustained neural discharge in monkey DLPFC neurons during the retention interval of delayed-response (DR) tasks suggested that this region is involved in on-line maintenance and manipulation of information (i.e., working memory). Functional neuroimaging of DLPFC revealed that activity in this region does not necessarily occur during the entire delay period of a memory-guided spatial delayed-response task, but instead at the time of the response selection (Rowe et al., 2000). Furthermore, delayrelated activity occurs in DLPFC only when a response can be selected at the beginning of the delay interval (Pochon et al., 2001).

Based on these results, Rowe et al. (2000) proposed a role of DLPFC for the selection of representations for upcoming actions, rather than the retention of sensory information. This interpretation fits with functional magnetic resonance imaging and transcranial magnetic stimulation studies showing that DLPFC is also involved in action selection tasks without a strict working-memory component.

Received Dec. 11, 2006; revised Jan. 7, 2007; accepted Jan. 7, 2007.

Correspondence should be addressed to Rogier B. Mars, Institute of Cognitive Neuroscience, University College London, 17 Queen Square, London WC1N3AR,UK.E-mail: r.mars@ucl.ac.uk.

DOI:10.1523/JNEUROSCI.5344-06.2007

Copyright $\odot$ 2007 Society for Neuroscience $\quad$ 0270-6474/07/271801-02\$15.00/0
This selection-dependent activity seems to be stronger when subjects freely select an action than when subjects respond to explicit movement cues. Furthermore, DLPFC displays a large flexibility in the type of information that can be processed. Although DLPFC is active during selection tasks regardless of modality, effective connectivity (i.e., the influence activity in one region has on activity in another region) between DLPFC and posterior brain areas depends on the type of processed information and on task demands (Egner and Hirsch, 2005; Rowe et al., 2005). These neuroimaging studies suggest that the dorsolateral prefrontal cortex might be involved in action selection and could play a specific role in top-down control of neural activity in regions processing taskrelevant representations. However, these imaging studies do not address the actual neural processing within this region.

A neurophysiological study by Procyk and Goldman-Rakic (2006), published recently in the Journal of Neuroscience, complements and further informs the neuroimaging results. In their experiment, macaque monkeys were required to perform two spatial tasks [Procyk and Goldman-Rakic (2006), their Fig. 1 (http://www.jneurosci.org/cgi/content/ full/26/44/11313/F1)]. In the first task, the DR task, monkeys were cued one of four spatial locations and required to execute a movement toward the cued target after a delay period during which there was no visual input. This task corresponds to the classical delayed-response paradigm. In the second task, the problem-solving (PS) task, the monkeys were not informed which of the targets would be required on any given trial. Instead, to be rewarded, the monkey had to determine the correct response by trial and error ("search" phase). After solving the task, the correct sequence had to be repeated three times ("repetition" phase). This combination of tasks allowed the authors to compare sustained neural firing in spatially selective DLPFC neurons during the "standard" delay period of a DR task and during the period between performance feedback and preparation of the next response during the search and repetition phases of the PS task.

The spatial selectivity of caudal DLPFC neurons expressing delay-related activity was determined during the repetition period of the PS task. Of the spatially tuned cells, the majority retained their spatial selectivity across tasks (PS and DR) and phases (search and repetition) [Procyk and Goldman-Rakic (2006), their Fig. $4 \mathrm{~A}$ (http://www.jneurosci.org/cgi/content/ full/26/44/11313/F4)]. To address whether the delay-period firing of these neurons reflected the coding of the past location to which the monkey moved or coded the upcoming movement location, activity during search trials was analyzed according to previous or upcoming responses [Procyk and Goldman- 
Rakic (2006), their Fig. 6A (http://www. jneurosci.org/cgi/content/full/26/44/ 11313/F6)]. Spatial selectivity of neuronal responses was only present in the case of upcoming responses, suggesting a predominance of a prospective coding of the upcoming movement, rather than retrospective coding of information.

This result fits nicely with selectiondependent activation of the DLPFC (Rowe et al., 2000) and explains the delayrelated activation reported in delayedresponse tasks. Furthermore, DLPFC neuronal firing was stronger during the search than during the repetition phase of the PS task at the end of and between trials [Procyk and Goldman-Rakic (2006), their Fig. 5 (http://www.jneurosci.org/cgi/content/full/26/44/11313/F5)], similar to the increased DLPFC activity found in imaging studies on free selection of actions.

During the PS task, population activity became progressively more spatially selective immediately after incorrect responses, in the first phase of the delay period, when a new response had to be selected [Procyk and Goldman-Rakic (2006), their Fig. 7 (http://www.jneurosci. org/cgi/content/full/26/44/11313/F7)] . Spatial selectivity became stronger until the first correct trial, when the monkey had identified the correct response. This modulation of DLPFC activity by task period led Procyk and Goldman-Rakic to suggest that "the adaptive properties of prefrontal delay activity are expected to reflect the basic mechanisms by which they influence connected structures" (Procyk and Goldman-Rakic, 2006). Again, this result complements neuroimaging studies, in which greater coupling between DLPFC and posterior brain regions can be seen during free selection of information, when the rule required for selection is unknown (Rowe et al., 2005). To select the correct information needed for the task at hand, DLPFC interacts with other task-specific areas that process specific stimulus-related information, potentially biasing processing in posterior areas to suit the task at hand.

Although the results from the present study seem to line up quite well with previous imaging studies, the precise mechanisms responsible for the increases in effective connectivity, as well as the evolution of neural activity in more posterior target areas, remain unknown. It would be interesting to see, at the neurophysiological level, the changes in neuronal activity during problem-solving tasks for nonspatial cues, and how cells in prefrontal and posterior areas synchronize as behavior progresses. Because Procyk and GoldmanRakic (2006) focused on a spatial task, a logical continuation would be to study these processes in nonhuman primates using different stimuli and different stimulus modalities. The experiments of Procyk and Goldman-Rakic (2006) provide a building block for such studies.

\section{References}

Egner T, Hirsch J (2005) Cognitive control mechanisms resolve conflict through cortical amplification of task-relevant information. Nat Neurosci 8:1784-1790.

Pochon JB, Levy R, Poline JB, Crozier S, Lehéricy S, Pillon B, Deweer B, Le Bihan D, Dubois B (2001) The role of the dorsolateral prefrontal cortex in the preparation of forthcoming actions: an fMRI study. Cereb Cortex 11:260-266.

Procyk E, Goldman-Rakic PM (2006) Modulation of dorsolateral prefrontal delay activity during self-organized behavior. J Neurosci 26:11313-11323.

Rowe JB, Toni I, Josephs O, Frackowiak RSJ, Passingham RE (2000) The prefrontal cortex: response selection or maintenance within working memory? Science 288:1656-1660.

Rowe JB, Stephan KE, Friston K, Frackowiak RSJ, Passingham RE (2005) The prefrontal cortex shows context-specific changes in effective connectivity to motor or visual cortex during the selection of action or colour. Cereb Cortex 15:85-95. 